\title{
Cell-free DNA
}

\section{An emerging technology in prenatal genetic screening}

Emily Pickering

\begin{abstract}
Chromosomal disorders arise from errors in cell division and many are detected during prenatal development. Prenatal genomic screening techniques involve invasive methods such as chorionic villus sampling and amniocentesis. In this feature, current invasive techniques for genetic screening will be examined in relation to the development of non-invasive prenatal technology. As cell-free fetal DNA methods continue to develop and be integrated into clinical practice, there is an opportunity for improvement in the detection and reliability of the screening process. In clinic, there are disparities between clinicians and patients surrounding both understanding of the processes and the discussion on the technique limitations. Noninvasive methods are continually being improved for detecting genetic disorders through the use of cell-free fetal DNA, and with these advancements, these processes will become safe, costeffective, and reliable for pregnant mothers when undergoing genetic screening and counselling.
\end{abstract}

\section{INTRODUCTION}

Chromosome disorders, involving portions of chromosomes altered by mistakes in cell division, are widely prevalent and affect approximately 1 in 250 people. ${ }^{1,2}$ During meiosis, nondisjunction occurs when two of the same sister chromatids or homologues fail to separate, resulting in doubling of the amount of genetic information in a single gamete. ${ }^{1}$ This can lead to trisomy or monosomy conditions such as Down syndrome and Turner syndrome respectively. ${ }^{1}$ During manipulation of the genome, microdeletions, rearrangements, or duplications can occur, causing a multitude of diseases including cancers and cardiovascular disease. ${ }^{3}$

Over recent decades, genomic screening techniques have improved with technological development. Existing methods for diagnosing chromosomal disorders involve karyotyping, wherein chromosomes are visualized for the detection of structural changes like whole or partial chromosome duplication or deletions. ${ }^{3}$ This method is reliable for diseases involving large chromosomal change (such as Down syndrome), but has difficulty elucidating small changes to the gene sequences. ${ }^{3}$ Alternatively, modern sequencing technologies effectively detect small changes in genes that can alter function, but are unable to elucidate larger-scale partial or whole chromosome abnormalities like Down syndrome. ${ }^{3}$ Currently, genome-wide genetic screening using next-generation methods can diagnosis children and adults with chromosomal disorders. ${ }^{3,4}$ Microarrays use oligonucleotide probes to perform large DNA sequence comparisons of normal genomes to genomes of disease states. ${ }^{3,4}$ These sequences provide information on certain genes, regulatory mechanisms, and pathways which are altered in the disease state, making them useful for the detection of multigene disorders or monogenetic disease, but not as useful for whole or partial chromosomal abnormalities. ${ }^{3,4}$ Other methods such as massive paired-end parallel sequencing allow genome sequencing to be simplified and become cost-effective for clinical use. ${ }^{4}$

Many disorder-causing genomic abnormalities can be elucidated from fetal DNA using an array of evolving genetic screening techniques, such as karyotyping for larger chromosome abnormalities, or microarrays and massive parallel sequencing for smaller genetic changes. ${ }^{3,4}$ Current prenatal screening involves the collection of maternal or fetal cells using invasive methods such as chorionic villus sampling or amniocentesis. ${ }^{5,6}$ Chorionic villus sampling involves taking cells from the outer membrane of the placenta, which are subsequently cultured for karyotyping and sequence analysis. Alternatively, amniocentesis involves sampling of the fetal tissue present in the amniotic fluid. ${ }^{5,6}$ Both methods involve inserting a needle into the pregnant mother, and often growth of the cultured cells cannot provide adequate DNA for the necessary genetic testing and the procedure must be repeated. ${ }^{5,6}$ Recollection of tissue samples from the placenta or amniotic fluid increases financial burden on the public Canadian health care system. Because of the invasive nature and potential downsides of chorionic villus sampling and amniocentesis, the future of safe, cost-effective screening for pregnant women will involve noninvasive prenatal screening. Recently, cell-free DNA methods have been rising as a means to provide the genetic information available in the placenta. ${ }^{4,7}$

\section{CELL-FREE DNA DISCOVERY AND IMPORTANCE OF NON- INVASIVE TECHNIQUES}

In 1997, the discovery of fetal DNA concentrations detectable in maternal plasma was important for propelling prenatal medicine., ${ }^{4,7}$ Unfortunately, the clinical utility of cell-free DNA (cfDNA) in the maternal bloodstream was limited to isolating genes from the paternal Y chromosome, until the development of PCR-based techniques and-more recently-next-generation sequencing., ${ }^{4,8}$ PCR-based sequencing methods are used reliably to diagnose single-gene disorders with cfDNA testing, but are not accurate for disorders with multiple causal factors. ${ }^{8,9}$ Even with multi-gene accuracy, it would be too time-consuming to use a PCR-based method to examine multiple genes. ${ }^{8,9}$ Consequently, the demand for a non-invasive prenatal technique for multi-gene analysis resulted in the development of next-generation sequencing. Next-generation sequencing provides greater sensitivity when 
diagnosing prenatal chromosome and genetic abnormalities, as it screens for many disease-causing mutations in a single assay, evaluating more mutation-prone DNA than earlier methods. ${ }^{8,9}$ One issue with invasive prenatal screening is that there is not always adequate amounts of DNA collected for all the screening techniques to be completed properly; however, recent advancement in parallel sequencing, PCR, and other next-generation methods allows for amplification of very small amounts of DNA for the necessary screening assays. ${ }^{6,9}$ Cell-free fetal DNA is only present in low amounts in the maternal bloodstream, creating difficulties in isolating and discerning maternal and fetal genes. ${ }^{4,79}$ In order to accurately measure fetal genomic information, a genetic marker is used-such as alleles found exclusively on the Y chromosome-to quantify the amount of fetal DNA present in the sample. ${ }^{8}$ For female fetuses, estimates must be made from a variety of single nucleotide polymorphisms (SNPs) since there are no unique chromosomes., ${ }^{910}$ SNPs account for approximately $90 \%$ of genetic information and can provide highly accurate diagnostic information through nextgeneration sequencing. ${ }^{10}$ Not only is next-generation sequencing highly accurate, it is timely and cost-effective, taking approximately twenty-four hours to complete screening of multiple genetic disorders in a single run., ${ }^{8,9}$

\section{NON-INVASIVE PRENATAL TESTING FOR CLINICAL DIAGNOSIS}

While non-invasive prenatal testing can assess the risk of inheritance for genetic disorders in a safe, cost-effective manner, there are limitations for use in the clinical setting. ${ }^{11}$ Despite the ability to successfully predict diseases like Down syndrome, noninvasive methods are poor diagnostic tools, and $42 \%$ of women with abnormal non-invasive test results proceed with confirmatory invasive testing. ${ }^{11}$ Better non-invasive technologies are needed to examine cfDNA and chromosomal aberrations, which are currently being missed in clinical use. ${ }^{12}$ Despite limitations, most pregnant women will opt for the non-invasive method as it poses less risk to the pregnancy. ${ }^{112}$ Breveglieri et al. showed that with technological advancement and methods like $\mathrm{dPCR}$ and next-generation sequencing, the increased sensitivity of non-invasive prenatal testing will improve its clinical viability. ${ }^{13}$ Additionally, non-invasive techniques present a significant financial burden on the public Canadian health care system, as it is expensive to fully sequence maternal plasma DNA. ${ }^{14}$ However, the input of massive parallel sequencing using select areas of the genome has made the process more cost-effective. ${ }^{14}$ The amount of maternal DNA that needs to be sequenced along with the fetal DNA to produce an accurate fetal DNA analysis needs to be determined in order to predict the clinical application cost. ${ }^{14}$ Until the balance of cost and the effectiveness of this method is met, non-invasive prenatal testing is important as a risk assessment tool rather than a diagnostic tool.

\section{ETHICS AND CONCERNS OF NON-INVASIVE PRENATAL SCREENING}

There are significant ethical concerns with non-invasive screening such as: informed decisions; access to the service; genetic counselling; and interpreting the test results. ${ }^{14}$ Since there exists a large push toward implementation of non-invasive methods in clinical settings worldwide, miscommunication between practitioners and patients create a divide on the accuracy and benefits of non-invasive testing. ${ }^{15}$ Currently, non-invasive testing is not fully understood as an assessment of chromosome disorder risk as clinicians continue to misdiagnose illness based solely on noninvasive methods. ${ }^{15}$ In Ontario, non-invasive screening is performed as a risk assessment for trisomy 21,13 , and 18 as it is accurate, quick, and provides minimal risk to the fetus. However, it requires invasive techniques or imaging modalities such as ultrasounds to confirm the presence or absence of these three chromosomal disorders. ${ }^{16,17}$ Until knowledge and technology progress enough to result in costeffective and reliable non-invasive screening, this method can only access risk and serves no benefit in the clinical setting as a lone diagnostic tool. ${ }^{15-17}$

\section{CONCLUSION}

Chromosomal disorders arise from errors in cell division and DNA replication. During prenatal diagnosis, the invasive methods of chorionic villus sampling and amniocentesis are used for a variety of fetal abnormalities. Cell-free fetal DNA analysis is developing and being utilized as a non-invasive technique. With new technology and next-generation sequencing, cfDNA methods are becoming more reliable for $\mathrm{Y}$ chromosome abnormalities, detecting trisomy conditions such as Down syndrome, and moving toward the detection of difficult genomic changes. Unfortunately, this tool is presented as a diagnostic method rather than a current risk assessment technique, leading to costly and unreliable clinic utility. As research and development of non-invasive prenatal testing continues, the procedure will become more reliable and cost-effective and will hopefully be able to diagnose prenatal genetic conditions without the need for confirmatory analysis.

\section{REFERENCES}

1. Genetics Home Reference. Are chromosomal disorders inherited? [Internet]. National Institutes of Health; c2019 [cited 2019 July 20]. Available from: https://ghr.nlm.nih.gov/primer/inheritance/ chromosomalinheritance

2. Moorthie S, Blencowe H, Darlison MW, et al. Chromosomal disorders: estimating baseline birth prevalence and pregnancy outcomes worldwide. J Community Genet. 2018 Oct;9(4):377-86. http://doi. org/10.1007/s12687-017-0336-2

3. Shaffer LG, Bejjani BA. Development of new postnatal diagnostic methods for chromosome disorders. Semin Fetal Neonatal Med. 2011 Apr;16(2):114-18. http://doi.org/10.1016/j.siny.2010.11.001

4. Page-Christiaens L, Klein H, editors. Noninvasive prenatal testing (NIPT): applied genomics in prenatal screening and diagnosis. Cambridge: Academic Press; 2018. Section 1, Cell-freeDNA (cfDNA): overview and technology, p. 1-48.

5. Chorionic villus sampling [Internet]. Mayo Clinic; c2019 [cited 2019 July 20]. Available from: https://www.mayoclinic.org/testsprocedures/chorionic-villus-sampling/about/pac-20393533

6. Amniocentesis [Internet]. Vancouver (BC): BC Women's Hospital; c2019 [cited 2019 July 21]. Available from: http://www.bcwomens. ca/our-services/pregnancy-prenatal-care/prenatal-diagnosticprocedures/amniocentesis

7. Lo YM, Corbetta N, Chamberlain PF, et al. Presence of fetal DNA 


\section{FEATURE ARTICLE}

in maternal plasma and serum. Lancet. 1997 Aug;350(9076):485-7.

https://doi.org/10.1016/S0140-6736(97)02174-0

8. Chitty LS, Mason S, Barrett AN, et al. Non-invasive prenatal diagnosis of achondroplasia and thanatophoric dysplasia: next-generation sequencing allows for a safer, more accurate, and comprehensive approach. Prenat Diagn. 2015 Feb;35(7):656-62. https://doi. org $/ 10.1002 /$ pd.4583

9. Liu X, Zhang C, Liu K, et al. Multiple SNPs detection based on lateral flow assay for phenylketonuria diagnostic. Anal Chem. 2018 Feb;90(5):3430-6. https://doi.org/10.1021/acs.analchem.7b05113

10. Neufeld-Kaiser WA, Cheng EY, Liu YJ. Positive predictive value of non-invasive prenatal screening for fetal chromosome disorders using cell-free DNA in maternal serum: independent clinical experience of a tertiary referral center. BMC Med. 2015 Jun;13(1):129. https://doi. org/10.1186/s12916-015-0374-8

11. Yang S, Lv J, Si Y, et al. Diagnostic differences between patients opting for non-invasive prenatal testing and patients having traditional prenatal diagnosis. Int J Clin Exp Pathol. 2018 May;11(5):2831-8.

12. Breveglieri G, D’Aversa E, Finotti A, et al. Non-invasive prenatal testing using fetal DNA. Mol Diagn Ther. 2019 Apr;23(2):291-9. https://doi. org/10.1007/s40291-019-00385-2

13. Lo YM, Chiu RW. Genomic analysis of fetal nucleic acids in maternal blood. Annu Rev Genomics Hum Genet. 2012 Sep;13:285-306. https:// doi.org/10.1146/annurev-genom-090711-163806

14. Minear MA, Alessi S, Allyse M, et al. Noninvasive prenatal genetic testing: current and emerging ethical, legal, and social issues. Annu Rev Genomics Hum Genet. 2015 Aug;16:369-98. https://doi. org/10.1146/annurev-genom-090314-050000

15. de Jong A, Dondorp WJ, de Die-Smulders CE, et al. Non-invasive prenatal testing: ethical issues explored. Eur J Hum Genet. 2010 Mar;18(3):272-7. https://doi.org/10.1038/ejhg.2009.203

16. Non-invasive Prenatal Testing [Internet]. Ottawa (ON): Prenatal Screening Ontario; c2019 [cited 2019 Oct 30]. Available from: https:// www.bornontario.ca/en/pso/about-prenatal-screening/non-invasiveprenatal-testing.aspx.

17. Audibert F, Bie ID, Johnson JA, et al. No. 348-Joint SOGC-CCMG guideline: update on prenatal screening for fetal aneuploidy, fetal anomalies, and adverse pregnancy outcomes. J Obstet Gynaecol Can. 2017 Sep;39(9):805-17. https://doi.org/10.1016/j.jogc.2017.01.032 